\title{
Ensemble Classifications for Student Academics Performance Data Set
}

\author{
Dr.G.Ayyappan, \\ Associate Professor, Bharath Institute of Higher Education and Research, Chennai \\ ayyappangmca@gmail.com
}

\begin{abstract}
The higher education institutes use data mining tools and techniques for academic improvement of the student performance and to prevent drop out. The data consists of socio-economic, demographic as well as academic information of three hundred students with 22 attributes. Five ensemble classification methods Attribute Selected Classifier, Bagging, Classification Via Regression, Weighted Instances Handler Wrapper and Multi Class Classifier were used. The Class Attendance Percentage attribute makes the highest impact in the final semester results of the students in our dataset. The results showed that MultiClassClassifier outperforms the other classifiers based on accuracy and classifier errors.
\end{abstract}

Keywords: Attribute Selected Classifier, Bagging, Classification Via Regression, Weighted Instances Handler Wrapper and Multi Class Classifier.

\section{Introduction}

Educational data mining is an emerging field in the area of data mining. In this competitive world, the educational setting also uses data mining tools to explore and analyze student performance, predict their results to prevent drop out and focus on both good and academically poor performers, feedback for the faculties and instructors, visualization of data and to have a better assessment of learning process. The quality of education needs to be improved and educational data mining is a tool for this improvement. Modern educational institutes need data mining for their strategy and future plans. Student's performance depends on various factors like personal, social, economic and other environmental ones[8, 9]. The top-level educational institutes' authorities may utilize the outcome of the experimental results to understand the trends and behaviors in students' performance which may lead to design new pedagogical strategies [10].

In this paper organizes section one has related works and brief introduction of this fields. In section two represents materials and methods, In section three describes results and discussions and the section four presents conclusion

II. Materials and Methods

The students' academic performance data set borrowed from https://archive.ics.uci.edu/ml/datasets/Student+Academics+Performance (It was evaluated based on academic and personal data collected from 3 different colleges from Assam, India). The dataset tried to find the end semester percentage prediction based on different social, economic and academic attributes 


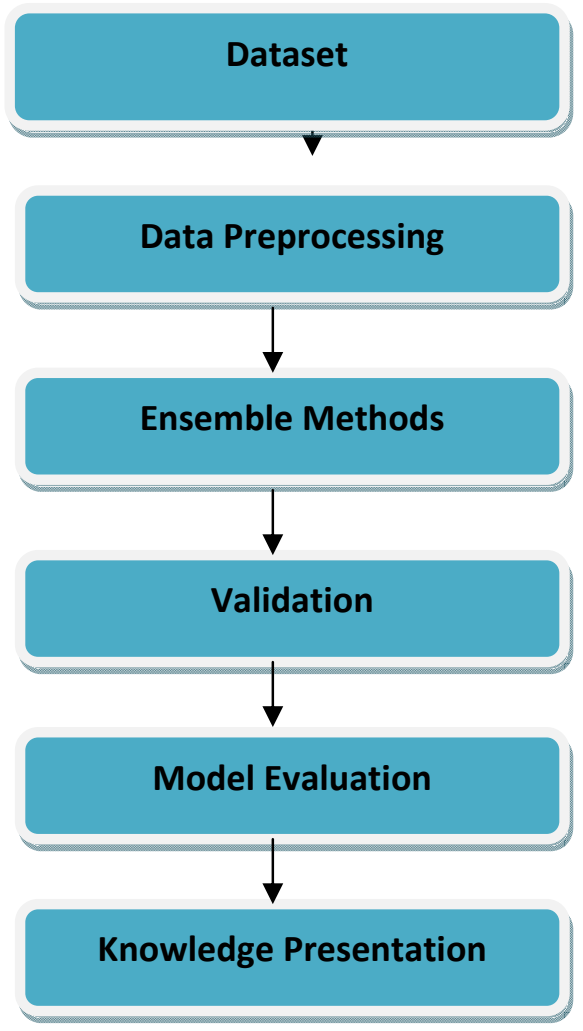

Figure 1 Architecture of Proposed Model

The stage is set for the experiments. WEKA has various classification algorithms. The authors had used AttributeSelectedClassifier, Bagging, ClassificationViaRegression, WeightedInstancesHandlerWrapper and MultiClassClassifier methods available in WEKA. These methods are supervised learning algorithms which use the training data to test the correctness of testing data.

Table 1 Attribute Description in the dataset

\begin{tabular}{|c|c|l|l|}
\hline S.No & Attribute & \multicolumn{1}{|c|}{ Description } & \multicolumn{1}{|c|}{ Values } \\
\hline $\mathbf{1}$ & GE & Gender & Male, Female \\
\hline $\mathbf{2}$ & CST & Caste & General,SC,ST,OBC,MOBC \\
\hline $\mathbf{3}$ & TNP & Class X Percentage & Best, Very Good, Good, Pass, Fail \\
\hline $\mathbf{4}$ & TWP & Class XII Percentage & Best, Very Good, Good, Pass, Fail \\
\hline $\mathbf{5}$ & IAP & Internal Assessment Percentage & Best, Very Good, Good, Pass, Fail \\
\hline $\mathbf{6}$ & ESP & End Semester Percentage & Best, Very Good, Good, Pass, Fail \\
\hline $\mathbf{7}$ & ARR & $\begin{array}{l}\text { Whether the student has back or } \\
\text { arrear papers }\end{array}$ & Yes, No \\
\hline $\mathbf{8}$ & MS & Marital Status & Married, Unmarried \\
\hline $\mathbf{9}$ & LS & Lived in Town or Village & Town, Village \\
\hline $\mathbf{1 0}$ & AS & Admission Category & Free, Paid \\
\hline $\mathbf{1 1}$ & FMI & Family Monthly Income in INR & $\begin{array}{l}\text { Very High, High, Above Medium, Medium, } \\
\text { Low }\end{array}$ \\
\hline $\mathbf{1 2}$ & FS & Family Size & Large, Average, Small \\
\hline $\mathbf{1 3}$ & FQ & Father Qualification & $\begin{array}{l}\text { IL, UM, 10, 12, Degree, PG (IL= Illiterate } \\
\text { UM= Under Class X) }\end{array}$ \\
\hline $\mathbf{1 4}$ & MQ & Mother Qualification & IL, UM, 10, 12, Degree, PG \\
\hline $\mathbf{1 5}$ & FO & Father Occupation & Service, Business, Retired, Farmer, Others \\
\hline $\mathbf{1 6}$ & MO & Mother Occupation & Service, Business, Retired, Farmer, Others \\
\hline $\mathbf{1 7}$ & NF & Number of Friends & Large, Average, Small \\
\hline $\mathbf{1 8}$ & SH & Study Hours & Good, Average, Poor \\
\hline
\end{tabular}




\begin{tabular}{|c|c|l|l|}
\hline $\mathbf{1 9}$ & SS & $\begin{array}{l}\text { Student School attended at Class X } \\
\text { level }\end{array}$ & Govt., Private \\
\hline $\mathbf{2 0}$ & ME & Medium & Eng,Asm,Hin,Ben \\
\hline $\mathbf{2 1}$ & TT & Home to College Travel Time & Large, Average, Small \\
\hline $\mathbf{2 2}$ & ATD & Class Attendance Percentage & Good, Average, Poor \\
\hline
\end{tabular}

\section{Results and Discussions}

In this section presents the ensemble classifiers applied in weka tool by using the student academic performance dataset. In this research work class value consider Class Attendance Percentage attribute. Testing options applied for Training set validation.

\begin{tabular}{|c|c|c|}
\hline S.No & Ensemble Classifier & Accuracy \\
\hline 1 & AttributeSelectedClassifier & $64.89 \%$ \\
\hline 2 & Bagging & $80.15 \%$ \\
\hline 3 & ClassificationViaRegression & $81.68 \%$ \\
\hline 4 & WeightedInstancesHandlerWrapper & $42.75 \%$ \\
\hline 5 & MultiClassClassifier & $91.60 \%$ \\
\hline
\end{tabular}

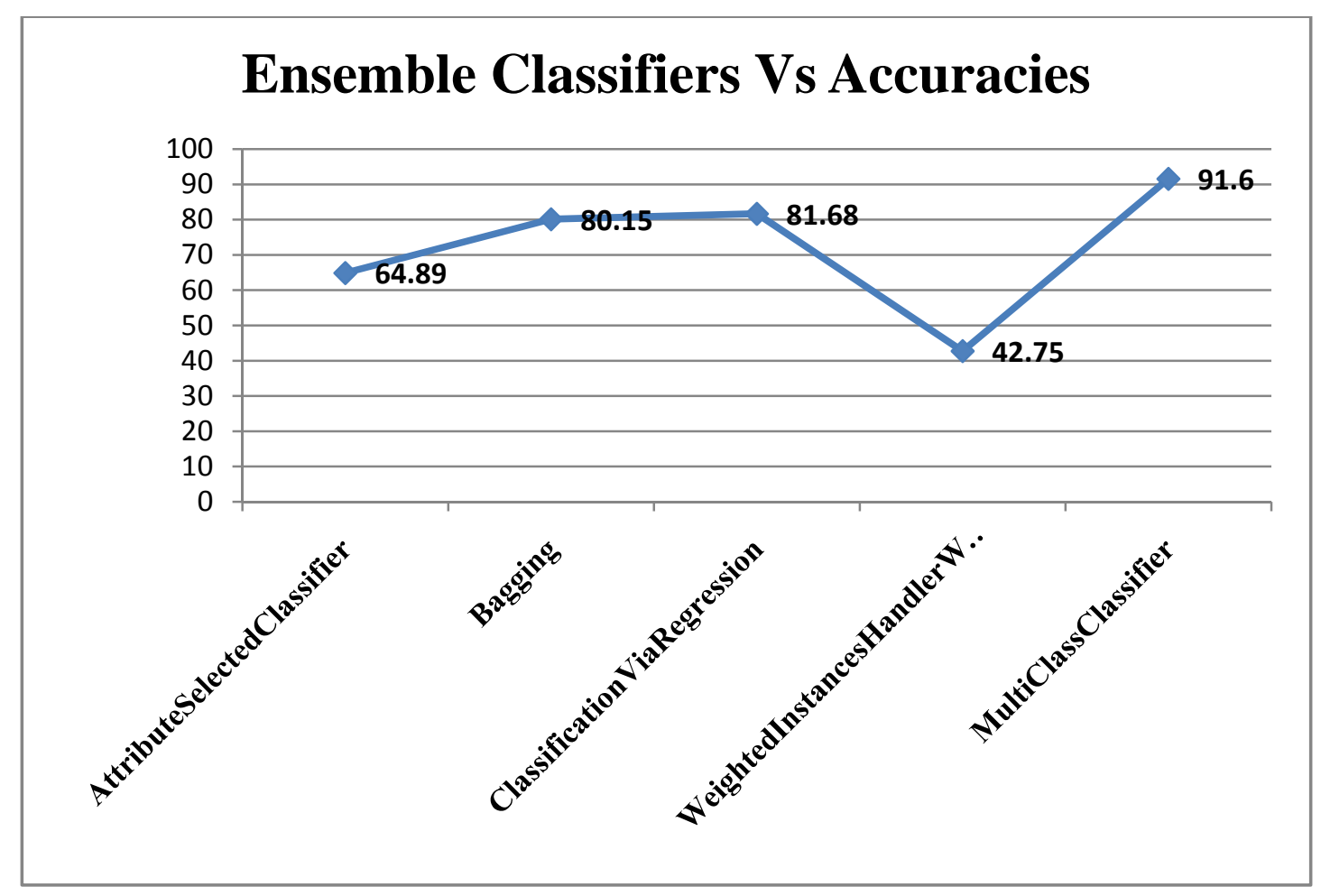

The above diagram clearly represents the meta classifications selected few algorithms namely AttributeSelectedClassifier has $64.89 \%$ accuracy level, Bagging has $80.15 \%$ accuracy level, ClassificationViaRegression has $81.68 \%$ accuracy level, WeightedInstancesHandlerWrapper has $42.75 \%$ accuracy level and MultiClassClassifier has $91.60 \%$ accuracy level. The Bagging, ClassificationViaRegression and MultiClassClassifier have more than $80 \%$ accuracy level.

\section{Conclusion}

The total numbers of records were 300 with 22 attributes. They were AttributeSelectedClassifier, Bagging, ClassificationViaRegression,WeightedInstancesHandlerWrapper and MultiClassClassifier. The data mining tool used in the experiment was WEKA 3.8.1 Based on the accuracy may conclude that the MultiClassClassifier Classification method was the most suited algorithm for the dataset. 


\section{References}

[1] Hussain S, Dahan N.A, Ba-Alwi F.M, Ribata N. Educational Data Mining and Analysis of Students ${ }^{\mathrm{TM}}$ Academic Performance Using WEKA. Indonesian Journal of Electrical Engineering and Computer Science. 2018; Vol. 9, No. 2. February. pp. $447 \sim 459$

[2] Amrieh, E. A., Hamtini, T., \&Aljarah, I. 2016. Mining Educational Data to Predict Student’s academic Performance using Ensemble Methods. International Journal of Database Theory and Application, 98, 119-136.

[3] Amrieh, E. A., Hamtini, T., \&Aljarah, I. 2015, November. Preprocessing and analyzing educational data set using X-API for improving student's performance. In Applied Electrical Engineering and Computing Technologies AEECT, 2015 IEEE Jordan Conference on pp. 1-5. IEEE.

[4] Hijazi, S.T. and S.M.M.R. Naqvi, Factors Affecting Students' Performance, A Case Of Private Colleges. Bangladesh e-Journal of Sociology, 2006. 3(1): p. 10.

[5] Bhardwaj, B.K. and S. Pal, Data Mining: A prediction for performance improvement using classification. International Journal of Computer Science and Information Security, 2011. 9(4): p. 5.

[6] Strecht, P., et al., A Comparative Study of Classification and Regression Algorithms for Modelling Students' Academic Performance. Proceedings of the 8th International Conference on Educational Data Mining, 2015: p. 3. 